\title{
Le transporteur mitochondrial UCP2: rôle dans l'immunité non spécifique et la production des ions superoxide
}

Les mitochondries jouent un rôle essentiel dans le contrôle des flux d'énergie dans les cellules. Ces organites assurent la respiration cellulaire, c'est-à-dire l'oxydation des substrats, notamment celle des co-enzymes réduits, NADH et FADH. L'oxydation de ces co-enzymes est un processus accroissant l'énergie libre disponible. En fait ce processus est couplé à la phosphorylation de I'ADP, mécanisme consommant de l'énergie, lui-même couplé au transport de protons. L'ensemble des deux mécanismes constitue la phosphorylation oxydative qui permet aux mitochondries d'être à la fois les principaux fournisseurs d'ATP et les principaux régénérateurs de coenzymes oxydés. En fait, le couplage entre la respiration mitochondriale et la phosphorylation de l'ADP n'est pas total. Une partie de l'énergie des oxydations n'est pas utilisée pour la synthèse d'ATP. De l'énergie est utilisée pour des transports à travers la membrane interne des mitochondries. Une autre fraction de l'énergie est perdue sous forme de chaleur, toute respiration étant accompagnée de production de chaleur. Les pertes d'énergie par les mitochondries résultent soit d'un manque d'efficacité des chaînes respiratoires, soit de fuites de protons à travers la membrane interne. Un cas particulier est celui des adipocytes bruns dont la membrane mitochondriale interne est équipée du transporteur de proton spécifique UCP1 (uncoupling protein). UCP1 découple la respiration et provoque la dissipation de la quasitotalité de l'énergie des oxydations sous forme de chaleur par le tissu adipeux brun des nouveau-nés de mammifère, des rongeurs qui n'hibernent pas et sont exposés au froid, ou encore de ceux qui hibernent lors de leur réveil [1-3].

\section{La famille des UCP}

En 1997, UCP2, un gène homologue de celui codant pour l'UCP du tissu adipeux brun fut identifié $[4,5]$. Ce gène est transcrit dans de nombreux tissus et types cellulaires tels les poumons, reins, tube digestif, tissus adipeux, épithéliums, macrophages. Sa forte expression, chez des souris résistantes à I'obésité lorsqu'elle sont soumises à un régime hyperlipidique, conduisit alors à proposer un rôle anti-obésité lorsqu'elles sont soumises à cette nouvelle UCP [4]. Si certains travaux de physiologie, biochimie ou génétique étaient en accord avec une telle hypothèse, plusi eurs autres études la remettaient en cause (pour revue voir [6] et [7]). Deux autres UCP furent identifiées, I'une chez les mammifères $[6,7]$, I'autre chez les plantes [8].

\section{Les macrophages des souris UCP2-} sont plus actifs

Afin de comprendre les rôles d'U CP2, notre laboratoire, en collaboration avec celui de Sheila Collins (Duke University Medical Center) et de Denis Richard (Université Laval, Québec) a produit des souris dont le gène UCP2 a été interrompu par recombinaison homologue et étudié leurs phénotypes [9]. Les souris mutantes résistent normalement au froid. Par ailleurs, elles ne sont pas obèses, même si elles consomment un régime hyperlipidique. Le gène UCP2 n'est donc pas impliqué dans la thermogenèse métabolique. L'obtention récente d'anticorps reconnaissant UCP2 nous permit de confirmer les données d'expression du transcrit, et de vérifier que la protéine était relativement abondante dans les organes lymphoïdes et les tissus riches en cellules effectrices de l'immunité. Ces résultats suggéraient que UCP2 puisse être impliqué dans la réponse immunitaire. Nous avons donc étudié la résistance des souris UCP2 $2^{++}$et $U C P 2^{-1-}$ à une infection chronique par Toxoplasma gondii. L'infection de souris normales par ce parasite provoque généralement la mort des animaux, ce qui fut vérifié chez toutes les souris UCP2 $2^{++}$. La surprise fut la constatation que toutes les souris déficientes pour le gène UCP2 survécurent à l'infection [9] indiquant donc une meilleure réponse immunitaire des souris mutantes. Les expériences in vitro démontrèrent alors que les macrophages des souris mutantes étaient capables d'éliminer le parasite avec très une grande efficacité.

\section{UCP2, mitochondries et radicaux libres}

En fait, comparés à ceux des souris de type sauvage, les macrophages des souris UCP2 $2^{-1}$ produisent plus d'espèces radicalaires de l'oxygène [9], molécules connues pour leur rôle essentiel dans la destruction des agents pathogènes. Le mécanisme de cette production excessive de radicaux libres n'a pas encore été entièrement élucidé. Par exemple, l'activité NADPH -oxydase des cellules n'a pas été mesurée. De même, la participation du monoxyde d'azote à ce phénotype reste à déterminer. On sait cependant que les mitochondries sont des sites importants de produc- 
tion de radicaux libres, et que la valeur du potentiel membranaire mitochondrial ou le niveau de couplage de la respiration à la phosphorylation de l'ADP module la production des radicaux libres mitochondriaux. Les macrophages sont des cellules riches en mitochondries. II semble donc, comme cela avait été proposé [10-12], qu'UCP2 soit un régulateur de la production des ions superoxide.

\section{L'absence d'UCP2: effet protecteur... ou délétère ?}

Il apparaît donc que l'absence d'UCP2 accroît la production des radicaux libres par les macrophages et confère aux souris une meilleure résistance aux agents pathogènes. Ces résultats indiquent qu'U CP2 peut moduler l'immunité innée et non spécifique et pourrait être une cible thérapeutique permettant de moduler l'activité des macrophages. Cependant, I'absence d'UCP2 dans des cellules autres que les macrophages (cellules épithéliales, neurones, cellules des îlots pancréatiques...) devrait logiquement aboutir aussi à une augmentation des taux de radicaux libres dans ces cellules. II reste à déterminer si dans ce cas, la moindre résistance aux stress oxydatifs pourrait être responsable de pathologies dégénératives.

En conclusion, il est probable qu'UCP2, en modifiant l'activité mitochondriale, a pour fonction principale d'empêcher l'élévation du taux des radicaux libres dans les celIules. UCP2 semble ainsi être un gène d'adaptation au métabolisme de l'oxygène et un gène de réponse aux oxydants.

1. Himms-H agen J, Ricquier D. Brown adipose tissue. In: Bray G, Bouchard C, James WPT, eds. New York: Marcel Dekker, 1997: 415-41.

2. Ricquier D, Bouillaud F. Les protéines découplantes mitochondriales. M ed Sci 1998; 14: 88997

3. Enerbäck S, Jacobsson A, Simpson E, et al. LP. Mice lacking mitochondrial uncoupling protein are cold-sensitive but not obese. N ature 1997; 387 : 90-4.

4. Fleury C, Neverova M, Collins S, et al. Uncoupling-protein-2: a novel gene linked to obesity and hyperinsulinemia. Nat G en et $1997 ; 15: 269-72$. 5. Gimeno R, Dembski M, Weng X, et al. Cloning and characterization of an uncoupling protein homolog. A potential modulator of human thermogenesis Diabetes 1997; 46: 900-6.

6 . Ricquier D, Bouillaud $F$. The uncoupling protein homologues: UCP1, UCP2, UCP3, StUCP and AtU CP. Biochem I 2000; 345: 161-79.

7. Boss $\mathrm{O}, \mathrm{H}$ agen $\mathrm{T}$, Lowell BB. Uncoupling Proteins 2 and 3 . Potential regulators of mitochondrial energy metabolism. Diabetes 2000; 49: 143-6. 8. Laloi M, Klein M, Riesmeier JW, et al. A plant cold-induced uncoupling protein. Nature 1997; 389: 135-6.

9. Arsenijevic D, O numa $H$, Pecqueur $C$, et al. Disruption of the uncoupling protein-2 gene in mice reveals a role in immunity and reactive oxygen species production. N at Genet $2000 ; 26: 345-9$.

10. Nègre-Salvayre $A, H$ irtz $C$, Carrera $G$, et al. A role for uncoupling protein-2 as a regulator of mitochondrial hydrogen peroxide generation. FASEB J 1997; 11: 809-15.

11. Skulachev, VP. Uncoupling: new approaches to an old problem of bioenergetics. Biochim Biophys Acta $1998 ; 1363$ : 100-24.

12. Diehl AM, Hoek JB. Mitochondrial uncoupling: role of uncoupling protein anion carriers and relationship to thermogenesis and weight control «the benefits of losing control ». J Bioenerg Biomembr 1999; 31: 493-506.

\section{Daniel Ricquier}

Cnrs UPR-9078, 9, rue Jules-H etzel, 92190 M eudon, France.
Identification d'un osmorécepteur. La pression osmotique systémique est probablement l'une des constantes de l'organisme qui est soumise au contrôle le plus strict. Sa détection est classiquement attribuée à des canaux ioniques sensibles aux modifications de la tension membranaire. Cependant, si de tels canaux ont été isolés chez les bactéries, la levure, C. legans ou la drosophile, aucun ne l'avait jusqu'à présent été chez les vertébrés. Le premier osmorécepteur des vertébrés vient probablement d'être identifié par l'équipe de Stephen Heller [1]. Les auteurs ont recherché une protéine homologue à deux autres canaux cationiques appartenant à la même famille: le premier, OSM-9, est exprimé chez la drosophile et impliqué dans la détection des stimulus mécaniques, et le second est le récepteur vanilloïde de type 1
(VR1) impliqué chez l'homme dans certains stimulus nociceptifs. Ils ont ainsi identifié chez le rat, la souris et I'homme un canal cationique qu'ils ont nommé VR-OAC (pour vanilloid receptor-related osmotically activated channel). La structure présomptive de VR-OAC, avec six domaines transmembranaires et trois motifs ankyrine à son extrémité amino-terminale, indique que cette protéine est bien un membre de la famille OSM9. VR-OAC est un canal cationique dont l'ouverture est activée par la diminution de la pression osmotique. II permet l'entrée de $\mathrm{Ca}^{2+}$ dans la cellule, provoquant la libération de $\mathrm{Ca}^{2+}$ intracellulaire. Sa sensibilité à I'hypotonicité est remarquable: il suffit en effet d'abaisser la pression osmotique à une valeur de $292 \mathrm{mosm} / \mathrm{kg} \mathrm{H} \mathrm{H}_{2} \mathrm{O}$, ce qui correspond à une diminution de $1 \%$ de sa valeur normale, pour observer une augmentation du $\mathrm{Ca}^{2+}$ intracellulaire. Chez le rat, VR-OAC est exprimé dans le système nerveux central par les neurones de certains organes circumventriculaires. On sait que ces neurones sont impliqués dans la détection des variations de pression osmotique systémique et se projettent vers les cellules neurosécrétoires des noyaux supra-optique et paraventriculaire de l'hypothalamus qui sécrètent... I'hormone antidiurétique. On le trouve également dans l'oreille interne et le rein, qui sont tous deux sensibles aux variations de pression osmotique ou hydrostatique, ainsi que dans certains neurones du ganglion trigéminé et les cellules de Merkel où ils pourraient jouer un rôle dans la sensibilité aux stimulus mécaniques.

[1. Liedtke W, et al. Cell $2000 ; 103$ : 525-35.] 